\title{
El papel de la educación en origen al abordar en la trata de jóvenes nigerianas hacia Europa \\ Esperanza Jorge Barbuzano ${ }^{1}$ Inmaculada Antolínez ${ }^{2}$
}

Recepción: 20-11-2017 / Aceptación: 15-01-2018

\section{Resumen}

Este artículo es resultado del proyecto de investigación "Trata de personas, salud integral y cuidados: mujeres transfronterizas en tránsito de Marruecos hacia Andalucía” de la Universidad Pablo de Olavide durante el año 2014. En Europa y, concretamente en España, la trata de mujeres de origen nigeriano cada vez más jóvenes e incluso menores, con fines de explotación - en este caso - sexual, es una realidad creciente. La investigación, desde un enfoque cualitativo y sustentado en observaciones, entrevistas y talleres de producción de Narrativas Creativas, analiza las condiciones en origen que inciden en la trata, prestando especial atención al papel de la educación formal y; por otro lado, presenta una experiencia de educación entre iguales. Los resultados nos arrojan que en la trata nigeriana hay condiciones de vulnerabilidad múltiple en origen donde la falta de formación es un factor esencial. Por otro lado, se destaca el potencial que tiene la sensibilización entre pares como acción para crear reflexión transformadora.

Palabras clave: Trata de seres humanos, educación, género, narrativas creativas.

\begin{abstract}
This article is the result of a research project called "People trafficking, integral health and care: cross-border women in transit from Morocco to Andalusia" from Pablo de Olavide University during the year 2014. In Europe and, concretely in Spain, the Nigerian women trafficking increasingly younger and even younger for exploitation purposes - in this case - sexual, is a growing reality. The research, based on a qualitative approach and supported on observations, interviews and Creative Narrative production workshops, analyze the conditions from its origin that influence the trafficking, paying special attention to the role of formal education and; on the other hand, it presents an educative experience between equals. The results show us that in Nigerian trafficking are conditions of multiple vulnerability in origin where the lack of training is an essential factor. On the other side, it highlights the potential that the sensitization between pairs has as an action to create a transformer reflection.
\end{abstract}

Key Words: trafficking in human beings, education, gender, creative narratives.

1. Máster Oficial en Escritura Creativa, Directora de Centro de Acogida, Fundación EMET Arcoíris, Córdoba, España; email: maspebeleten@gmail.com

2. Doctora en Ciencias Sociales, Profesora asociación-investigadora coordinadora, Universidad Pablo de Olavide, Sevilla, España; email: iantdom@upo.es 


\section{Introducción}

Durante siglos, millones de personas esclavas fueron desplazadas desde el África Occidental para abastecer de mano de obra gratuita a las potencias colonizadoras europeas. Este comercio fue abolido en el siglo XIX, aunque en la práctica continúa la trata de personas como la "esclavitud del siglo XXI". Esta fue definida por Naciones Unidas (2000), implicando unos actos tales como la captación, el transporte, el traslado, la acogida o la recepción de una persona; unos medios como uso de la fuerza o amenazas u otras formas de coerción, secuestro, engaño, abuso de poder o de una situación de vulnerabilidad y; unos fines que son la explotación de la persona bien sea sexual, laboral, trabajos forzados, esclavitud, servidumbre o extracción de órganos, así como el matrimonio forzado.

Si bien es cierto que la trata es un fenómeno global, queremos señalar los tres puntos de partida que explican la elección de esta investigación con mujeres, de origen nigeriano, mayormente jóvenes.

En primer lugar, aunque la trata de seres humanos se dé en todo el mundo, hay ciertas regiones que destacan como territorios de origen. Las víctimas detectadas en el Estado español con fines de explotación sexual, en año 2014, tenían como país de procedencia Rumanía (32\%), Nigeria (27\%) y República Dominicana (5\%); Brasil (5\%), Bulgaria (4\%) y Paraguay (4\%) (MSSSI, 2015). Según Eurostat (2015), a nivel europeo, la nacionalidad nigeriana sobresale, de entre el grupo de mujeres que son víctimas de trata, cuya procedencia es no europea. Por otro lado, es importante tener en cuenta la cuestión de género. De las víctimas o posibles víctimas que se calculan a nivel mundial, el 51\% son mujeres y el 20\% niñas (UNODC, 2016). Este rostro feminizado de la trata cobra especial dimensión cuando el fin de explotación es el sexual, aumentando el porcentaje al $96 \%$. A lo que se añade el tercer y último punto de partida que, recogiendo datos de Naciones Unidas, apunta a que en torno a un cuarto de las víctimas detectadas son menores de edad.

Esta presencia relevante de mujeres y jóvenes de Nigeria vinculadas a la trata en el Estado español y en Europa cobra especial fuerza a partir de la década del 2000, pudiéndose encontrar desde dicha fecha múltiples estudios por parte de organismos internacionales (UNODC, 2016, 2014, 2012, 2009; UNESCO, 2006; OIM, 2006; UNICRI, 2003; UNICEF, 2000). En la academia, la temática ha vivido un incremento de producción en la última década en el contexto español con trabajos sobre la relación entre trata y prostitución (Acién y Checa, 2011; Solana, 2011; Acién, 2010); así como migración y trata de personas (Jorge y Antolínez, 2015; Flamtermesky, 2014).

En el caso de la realidad nigeriana, la trata de jóvenes con fines de explotación sexual está insertándose en un cuerpo de trabajos propios, pudiendo destacarse los trabajos de Jorge, Antolínez y Cordero (2017); Hadjab (2016), Acién (2015), García de Diego (2014) 
o Rodríguez (2014). Entre los temas principales que han sido abordados se encuentran: la cuantificación del fenómeno; el papel del vudú en la coacción de las víctimas (Dols, 2013; Nwogu, 2008; Van Dijk, 2001); o las violaciones de derechos humanos (Women's Link Worldwide, 2014; Defensor del Pueblo, 2012). Sin embargo, no son tan abundantes estudios sobre las características del contexto de origen para explicar la trata nigeriana.

El trabajo de Women's Link Worldwide (2014) o el de Rodríguez y Sánchez (2013), evidencian el papel de las desigualdades económicas o la violencia intra-familiar $\mathrm{y}$ estructural como factores de importancia. El Informe de UNICRI (2003), también ahonda en las características culturales y familiares que favorecían a principios del año 2000 la migración hacia Italia y, las tesis de García de Diego (2014) y Hadjab (2016), abordan varias cuestiones sobre origen desde la revisión bibliográfica.

Este estudio parte de los resultados de Omorodion (2009), en cuyo estudio plantea que la etapa de secundaria es la de mayor vulnerabilidad a la captación en trata; así como de Hadjab (2016) que retrata entre las nuevas generaciones de migrantes a niñas nigerianas. Por ello, este trabajo tiene como primer objetivo interrogar el papel que juega la educación formal en origen en la temática. En segundo lugar, a partir del análisis de una experiencia de sensibilización entre pares realizada en la investigación en contextos educativos nigerianos, se reflexiona sobre la utilidad de estas acciones en la prevención de la trata de seres humanos.

\section{Metodología}

La metodología que se elaboró toma rostro de etnografía multisituada feminista (Gregorio, 2017; Marcus, 2001). Por el carácter multisituado, en etapas previas (2014-2015) se ha realizado trabajo de campo en el Estado español y en Marruecos, aunque dichos datos no son objeto de este artículo. En el caso de Nigeria, el trabajo de campo se realizó en agosto del año 2015. Los contextos de investigación fueron la ciudad de Calabar en el Estado de Cross River (principal territorio de trata interna); y Benin City en el Estado de Edo (principal Estado del que migran las jóvenes hacia Europa vinculadas a la trata externa).

En el diseño de la investigación se partió de un problema preliminar, esto es, la trata de mujeres y niñas nigerianas hacia España con fines de explotación sexual, que se convirtió en una cuestión de naturaleza sustantiva (Hammersley y Atkinson, 2003). Esta se interrogaba sobre las características que se dan en origen y en relación con la salida de mujeres y menores en redes de trata, prestando especial atención al papel de la educación por la significatividad de la llegada de menores en edad escolar. Dicha pregunta se vio complementada por "el mandato" que desde la praxis recibimos de las mujeres con las que habíamos trabajado previamente en Marruecos. Éstas, al saber que se viajaría a Nigeria, propusieron: "cuéntenles a las chicas allá en Nigeria la verdad del trayecto" (Mujer nigeriana, Casablanca, 2015). 
Se incorporó este reto al proceso de investigación, proponiendo a las mujeres en España y Marruecos que, quienes quisieran, enviaran un mensaje a las jóvenes nigerianas que estaban en origen, utilizando el vídeo como soporte y respetando las medidas de protección y anonimato. Sus propuestas pasaban por denunciar el trato recibido, describiendo episodios de violencia, subrayando los engaños o informando de la explotación que vivían. Con dicha decisión, se partió de una disolución de fronteras entre "sujeto-conocedor" y "objeto-de conocimiento "(Gregorio, 2017); reconociendo de esta manera a los jóvenes como sujetos activos del proceso de investigación y, en este caso, también de acción.

Por ello, la segunda pregunta diseñada fue conocer qué respuesta ofrecía el alumnado de secundaria, en tanto que colectivo diana para la captación en trata, a los testimonios de sus iguales sobre las condiciones del trayecto migratorio y la explotación en destino. A partir de dichas preguntas se pre-diseñaron dos bloques temáticos que articularon las herramientas de investigación: 1) cuestiones claves en origen para la trata $y, 2$ ) respuesta a propuestas de sensibilización entre pares.

Para asegurar la validez de la información, se realizó una triangulación tanto de investigadoras como de técnicas (Denzin, 1970). Se optó por el uso de tres técnicas de producción de datos: análisis documental, entrevistas y talleres de creación de Narrativas Creativas. Por otro lado, por el carácter del trabajo, la validación ética (Merriam, 1988) consistió en que las investigadoras se presentasen como intelectuales europeas, activistas contra la trata de personas que, además de producir información, partían del compromiso de realizar acciones para la prevención a partir de la demanda expresada por los sujetos de investigación. Se llevaron a cabo trece entrevistas (ocho asociaciones que trabajan con jóvenes en la prevención para trata; dos informantes claves del ámbito académico; la directora de la Agencia Nacional para la Prohibición de la Trata de Personas en Benin City; una enfermera del hospital público en Calabar y la directora de un centro educativo de secundaria en Calabar) y; cuatro talleres en centros de secundaria: tres mixtos y uno femenino.

El total de participantes en dichos talleres fue de 145, de los cuales 32 eran de sexo masculino y 113 , femenino. Las temáticas planteadas en los guiones de entrevistas se articulaban en torno a la pregunta 1 de la investigación (cuestiones claves en origen haciendo especial énfasis en la educación/escolarización); mientras que las temáticas abordadas en los talleres para facilitar el discurso creativo se centraron en la pregunta 2 (respuestas a herramientas de sensibilización), aunque en ellos se registró información pertinente para la pregunta 1.

La propuesta de los talleres partía de crear espacios para la producción de Narrativas Creativas (verbal, visual, audiovisual y corporal) con el objetivo de elaborar narraciones contextualizadas, que se originaran en las propias experiencias y que se dirigieran hacia 
la creación de significados individuales y sociales (Gandarias y García, 2014). El formato de taller constaba de una primera parte de calentamiento corporal; la realización de una ficha donde se dibujaban a sí mismas, sus dolores y formas de cuidados (introspección); el visionado del vídeo de sensibilización (momento dialógico) y, finalmente, el alumnado participaba ofreciendo sus respuestas al diálogo tanto de forma oral como por escrito. En una de las escuelas mixta también se dedicó una sesión a registrar el imaginario de género, a partir del dibujo de una silueta de hombre y de mujer, a la que añadieron rasgos físicos, de carácter, profesiones y tareas dentro y fuera del hogar.

Tras la transcripción de los datos, se sistematizó la información en tablas a partir de los temas de investigación. Siguiendo un proceso inductivo desde la Teoría Fundamentada (Glaser y Strauss, 1967), se revisaron algunas entrevistas y talleres de forma aleatoria tratando de identificar los subtemas de interés definiendo algunas categorías y las relaciones entre ellas (Creswell, 2013). Ambas investigadoras lo hicieron de forma independiente, poniendo en común aquellas categorías ante las que hubiera discrepancias, logrando el consenso en todas ellas (Escobar y Cuervo, 2008). Para facilitar el análisis, se cuantificaron los datos narrativos para obtener la prevalencia de las categorías en los discursos de entrevistas y talleres.

\section{Resultados}

A partir del análisis realizado, se seleccionaron categorías en relación con las preguntas de investigación conforme a una codificación axial (Charmaz, 2005) (Tabla 1).

\section{Condiciones en origen: ser hija, niña y mu- jer empobrecida en Nigeria}

El análisis sobre los factores en origen que influyen en que la trata sea endémica en ciertas partes de Nigeria, apunta a una situación de vulnerabilidad múltiple de las mujeres en el Estado de Edo, donde se encuentra Benin City. Un primer factor explicativo surgido de las entrevistas (6.6\%) fue la importancia de las pautas culturales y de parentesco.

En otras culturas nigerianas las niñas son vistas igual que los niños, y si me caso fuera sigo perteneciendo a mi familia de origen. Pero en Benin encuentras que hay una Ley de primogenitura y herencia. Es el niño varón el que es valorado aquí. Y eso nos viene justo de arriba, del palacio del Oba de Benin (E- K. E. Antropóloga, Benin City, 2015).

Tal y como explican Ugiagbe, Eghafona y Omorogiuwa (2007), la primogenitura y la patrilinealidad conllevan que la herencia del padre la reciba el primogénito varón, dejando a la esposa, así como a las hijas, en una situación de desprotección. De ahí la importancia del sistema de parentesco patrilineal y patrilocal que se mantiene en Benin City, y que coloca a las mujeres en un lugar del sistema por 
Tabla 1. Pregunta de investigación, categorías y frecuencia

\begin{tabular}{|l|l|c|}
\hline \multicolumn{1}{|c|}{ Pregunta de investigación } & \multicolumn{1}{|c|}{ Categorías } & Porcentaje \\
\hline \multirow{4}{*}{ Condición de origen } & Educación & $46.6 \%$ \\
\cline { 2 - 3 } & Situación económica & $26.6 \%$ \\
\cline { 2 - 3 } & Desigualdad de género & $20 \%$ \\
\cline { 2 - 3 } & Sistema de parentesco & $6.6 \%$ \\
\hline \multirow{4}{*}{ Respuesta acción sensibilización } & Responsabilidad de actores & $39.7 \%$ \\
\cline { 2 - 3 } & Causas de la trata & $38.3 \%$ \\
\cline { 2 - 3 } & Propuestas & $21.9 \%$ \\
\hline
\end{tabular}

debajo de sus compañeros varones. Por otro lado, en un $20 \%$ de los datos de entrevistas y talleres se apuntaba a que esta vulnerabilidad se ve reforzada por el propio proceso de socialización, donde "la niña crece sintiendo que ella es la responsable de los hombres en su vida: su padre, sus hermanos y más tarde su marido" (E- Coordinadora ONG GPI, Benin City, 2015).

A partir de los datos de campo, se planteó recoger en fichas la imagen que el alumnado tenía sobre qué es ser hombre y mujer. En relación con las profesiones, destacaron como mayoritarias en los hombres: futbolista y banquero, ambas reflejo del triunfo social. En el caso de las mujeres: abogada, enfermera, peluquera y profesora, donde el cuidado social o individual está presente. De entre las profesiones que apuntan exclusivamente a mujeres, vuelve a reforzarse el ámbito de los cuidados tanto en el espacio doméstico como social: peluquera, cocinera, lavandera y lavaplatos. En el caso de los trabajos exclusivos para hombres, mostrando escenario de poder están: presidente, gobernador, predicador y jefe.
Las explicaciones discursivas que ofrecieron sobre esta división laboral partían de las capacidades diferentes entre ambos sexos-géneros: "El motivo por el que creo que una mujer no puede ser presidenta de este país es porque la mujer no puede dirigir este país como lo haría un hombre" (Alumno escuela AKIM, Calabar, 2015). Esta construcción social desigual conlleva, igualmente, un tratamiento diferenciado a la hora de migrar.

Los tratantes dicen que no facilitarán a un chico viajar a menos que le pague todo el dinero por adelantado. En ese punto actúa como un traficante. Pero cuando asisten a chicas será cuando llegue a Europa cuando comience a devolver una cierta cantidad de dinero (E-Coordinadora GPI, Benin City, 2015).

Según datos de campo, las deudas que estas niñas y mujeres pueden pagar oscilan entre 20,000 a 80, 000 euros; mientras que el dinero que un chico paga por adelantado puede rondar los 3,000 a 6, 000 euros.

Estas cantidades han de ser tenidas en cuenta en un contexto donde el empobrecimiento, 
según los datos analizados (26.6\%), es una de las variables fundamentales que provocan las migraciones en situación de riesgo. Nigeria es uno de los países más ricos en petróleo de África, pero a su vez tiene un Índice de Desarrollo Humano definido como bajo con 0.527, ocupando el lugar 152 de 188 en el mundo (PNUD, 2016). A ello se unen las consecuencias que los Programas de Ajuste Estructural, impuestos en la década de los años ochenta, tuvieron en un gobierno con alto crecimiento por el petróleo; y que pasó a una situación de crisis estructural: "antes la gente salía a estudiar y volvía. A mediados de los años ochenta, el gobierno dejó de proveer de servicios sociales y las cosas se pusieron realmente difícil" (E-Coordinadora GPI, Benin City, 2015).

Unido a ello, la entrada del petróleo cambió también formas de vida tradicionales donde la familia extensa era red y cuidado social. Sin embargo, "ahora está ganando peso la familia nuclear (...) todos los estados entraron en el petróleo, y entró dinero libre. El problema continúa y no hay más trabajos en las compañías petrolíferas del gobierno, y las familias ya están obviando su responsabilidad social" (EJ. Charles. Antropólogo, Calabar, 2015). Por último, un tema de especial interés para la investigación era conocer qué sucedía en las etapas educativas en Nigeria para que se diera un incremento en la llegada de menores, tal y como se aprecia en el $46 \%$ de datos de entrevistas y talleres.
En la franja de edad entre 12 y 16 años, correspondiente a la etapa de secundaria, la vulnerabilidad aumenta, ya que es una edad "propicia" para la explotación, tanto por demanda juvenil de mercado en Europa, como por ser una etapa que supone un pequeño coste: "No podemos decir que la escuela secundaria sea totalmente gratis. Tienen que pagar una pequeña cantidad. Sabemos que algunas no lo pueden pagar porque son huérfanas o no tienen a nadie" (E- Directora escuela AKIM, Calabar, 2015).

Por ello, es igualmente una etapa susceptible de engaño ya que: "una de las mentiras que les cuentan para captarlas es la posibilidad de cursar estudios o tener trabajo en el extranjero" (E- Coordinador ACHF, Calabar, 2015). De ahí que, como recogen las entidades especializadas: "los tratantes se centran ahora más en zonas rurales donde el nivel de pobreza es alto, el de analfabetismo alto y el nivel de información bajo" (E-Coordinador Idia, Benin City, 2015).

La tasa de alfabetización de personas adultas en Nigeria es del $51.1 \%$, a lo que hay que sumarle que el trabajo infantil se sitúa en el 24.7\% (UNICEF, 2015). Tal y como apuntaba una de las entrevistadas, "tenemos una falta de formación como uno de los problemas que nuestras mujeres confrontan" (E-enfermera, Calabar, 2015); y la falta de un título en Nigeria reduce notablemente las expectativas de una vida digna. Por último, la convergencia entre coste de la educación y desigualdad de género implica, además, que si una familia 
tiene que escoger qué miembro continúa escolarizado normalmente será un hijo varón, mientras la niña se queda en casa o, en otras ocasiones, migra en el marco de las redes.

\section{Sensibilización entre pares: diálogo en- tre iguales}

Durante la primera fase del trabajo realizado en el Estado español y en Marruecos, una de las mujeres planteó: "Yo no quiero que ninguna chica africana tenga que vivir lo que yo he vivido, por eso cuento lo que he pasado. Para que ellas sepan la verdad" (Joven nigeriana, España, 2015). El conjunto de este y otros testimonios dio lugar a un pequeño vídeo, proyectado en cuatro centros de secundaria que provocaron las respuestas recogidas en la figura 1.

De entre los temas recurrentes aportados, se sistematizaron tres fundamentales: responsabilidad en la trata; causas de la trata y propuestas de salida o prevención.
En términos absolutos, la responsabilidad de los gobiernos en la temática (señalando mayormente la dejación de los mismos); la posibilidad del retorno de estas jóvenes ante el trato que se les está dando en Europa "como esclavas"; y, el papel de los familiares fueron las tres opciones más señaladas. Estas preocupaciones se interpretaron desde un enfoque de cuidados amplio (Coello y Pérez de Orozco, 2013), a partir del cual las menores conciben a los Estados como los principales garantes (en este caso, en dejación de funciones) de la salvaguarda y protección de las personas que están en su territorio; señalan la sociedad en su totalidad como red protectora que debería acoger a estas mujeres como miembros de una comunidad amplia. De ahí que señalan el retorno como solución al fallo de comunidad global.

Por último, aparece el papel de la familia ya que, desde ese cuidado amplio, tiene gran fuerza la corresponsabilidad parental, debili-

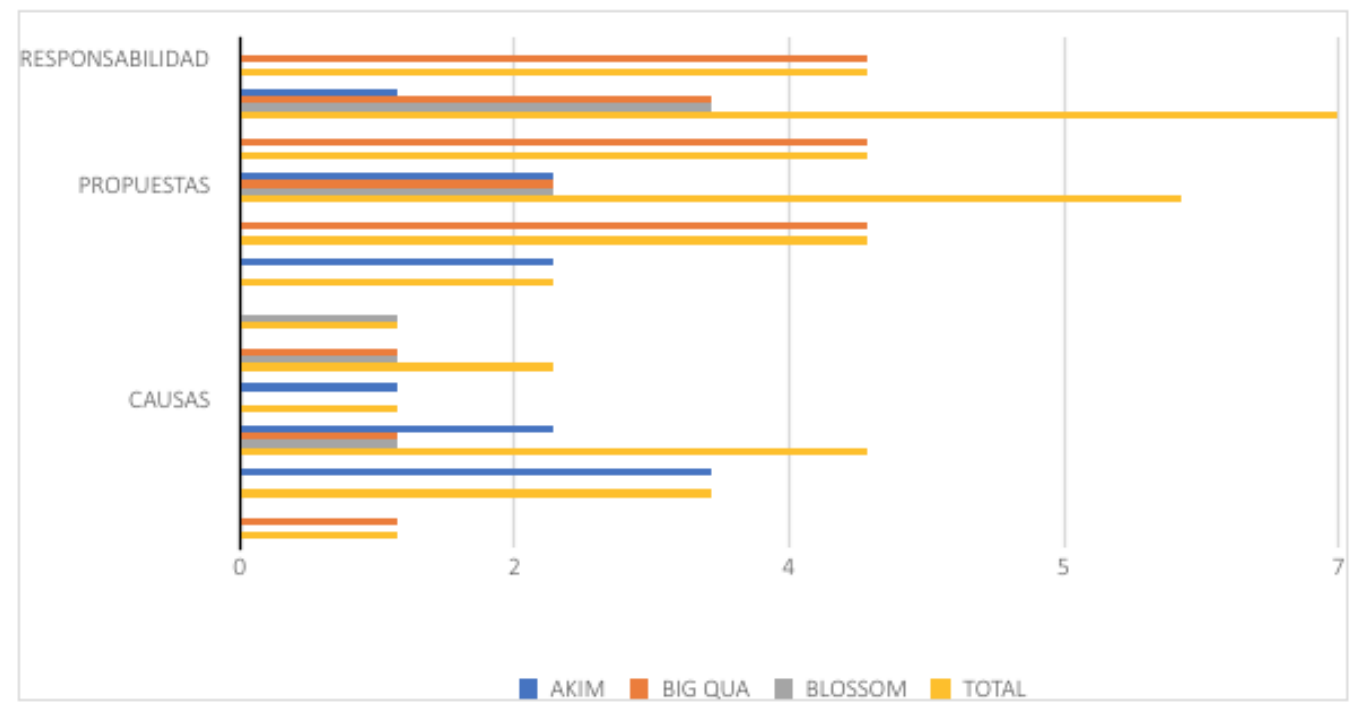

Figura 1. Temas con mayor prevalencia en comentarios del alumnado. 
tada ante la presión económica, la desprotección estatal y el cambio de modelo de familia. Unido a ello, señalan entre las causas la desigualdad de género, la pobreza y la falta de educación y formación crítica, en coherencia con las cuestiones abordadas previamente. Una alumna lo resumía de la siguiente forma:

Estoy segura que esas niñas no pudieron recibir educación. Yo creo que eso, la pobreza, la falta de cuidado parental son los motivos para que estas niñas se vayan (...) y la influencia también de sus iguales, como unas alumnas aquí que se fueron a Ghana para prostitución (Alumna, escuela AKIM, Calabar, 2015)

Las condiciones de desigualdad en Nigeria están empujando a multitud de niñas y jóvenes a migrar dentro de redes de trata. Las respuestas que ofrecieron al vídeo presentado evidenció la capacidad concientizadora que el diálogo entre iguales transcontinental puede tener; en tanto que estrategia para acceder a información real sobre la migración y la trata. Por otro lado, también como herramienta para favorecer la reflexión sobre propuestas de acción, tales como plantear el retorno; cuestionar la existencia de derechos para personas no ciudadanas en España; ofrecer cursos de formación a las mujeres para salir de esa situación o propuestas en el propio contexto escolar de origen: "Nosotras somos las generaciones más jóvenes y nos podríamos ver envueltas en lo mismo. Por eso creo que deberíamos hacer un seminario para crear conciencia. Afrontar estos problemas, y pararlo" (Alumna, escuela Big Qua, 2015).
El hecho de que hayan sido jóvenes como ellas y no mujeres blancas europeas las que les hablaran fue considerado clave para que el mensaje fuera legitimado por ellas y; por tanto, recibido de forma exitosa. En síntesis, por todo ello, se plantea la sensibilización entre pares como una herramienta de interés en la acción social sobre este fenómeno complejo y transnacional como es la trata de seres humanos desde África hacia Europa. Igualmente, se señalan los centros educativos como escenarios clave para crear espacios de reflexión permanente, donde el alumnado sea protagonista de la construcción de pensamiento crítico y propuestas de acción contra la trata de seres humanos.

\section{Discusión}

En este artículo se abordaron las condiciones en origen que inciden en la trata desde ciertas regiones de Nigeria, prestando especial atención al papel de la educación y; por otro lado, la respuesta a una experiencia de sensibilización entre iguales. De ello, se señalan tres aspectos como esenciales:

El primero es la importancia de un enfoque interseccional (Crenshaw, 1991) a la hora de analizar la trata, donde las condiciones de origen, la desigualdad de género, un sistema de parentesco que favorece dicha desigualdad, el empobrecimiento y la falta de acceso a la educación interactúan para colocar a las mujeres en una situación de absoluta exposición a la trata. Dicho contexto de partida ya ha sido abordado en informes previos (UNICRI, 2003; OIM, 2006; Women’s Link World- 
wide, 2014); aunque es importante señalar, también, trabajos como el de Kempadoo, Sanghera y Pattanik (2005) o Stock (2011) en el que, además de visibilizar esta vulneración de derechos, plantean las estrategias que ponen en marcha las mujeres para confrontarlo. Para el caso de este estudio, estas pasan por contar su historia, quienes están en España o Marruecos, y por las propuestas de las menores de secundaria para la prevención en trata.

En segundo lugar, esta desigualdad conlleva un mayor abandono escolar en niñas que en niños, tal y como han puesto en evidencia Tuwor y Sossou (2008) en Nigeria, Ghana y Togo. Además, Omorodion (2009) apunta a que la vulnerabilidad a la trata en secundaria está en relación con el empobrecimiento, desempleo, analfabetismo y bajo estatus en coherencia con lo mostrado en este trabajo. De ahí la importancia de fortalecer líneas de estudio que se centren, especialmente, en el papel de la escolarización y la etapa de secundaria como espacios de captación y prevención.

Por último, se concluye que apuestas metodológicas que parten del reconocimiento legítimo de los sujetos vulnerados como portadores de conocimiento, tal como plantearon Moll et al. (1992) en procesos educativos y comunitarios con poblaciones hispanas en Estados Unidos, pueden ser de gran utilidad en el abordaje de la trata. Trabajos como los de Flamtermesky (2014) avanzan en este posicionamiento donde se apuesta por la creatividad o el arte como herramientas útiles tanto en la investigación como - en este caso - una acción de sensibilización con jóvenes, a partir de un diálogo transnacional con sus iguales.

\section{Referencias}

Acién, E. (2015). Trabajadoras sexuales nigerianas en el poniente almeriense (Tesis doctoral). Universidad de Almería, España

Acién, E. y Checa, F. (2011). La actualidad del abordaje de la trata de personas para la prostitución forzada en España. El Plan Integral y sus implicaciones para trabajadoras del sexo inmigradas. Gazeta de Antropología, 27 (1)

Charmaz, K. (2005). Grounded theory in the 21st Century. En Denzin, N. K. \& Lincoln, Y. S. [Eds.] The SAGE handbook of qualitative research. London: Sage Publication

Crenshaw, K. W. (1991) Mapping the Margins: Intersectionality, Identity Politics, and Violence against Women of Color. Stanford Law Review, 43 (6), pp. 1241-1299

Creswell, J. W. (2013). Qualitative Inquiry and Research Design: Choosing Among Five Approaches. Washington, DC: Sage 
Coello, R. y Pérez de Orozco, A. (2013). Cómo trabajar la economía de los cuidados en la cooperación para el desarrollo. Agencia Andaluza de Cooperación Internacional al Desarrollo. Sevilla

Defensor del Pueblo (2012). La trata de seres humanos en España: víctimas invisibles. Defensor del Pueblo. Madrid

Denzin, N.K. (1970). Sociological Methods. A Sourcebook. Chicago, IL: Aldine Publishing Company

Dols, A. (2013). El vínculo entre el vudú y el delito de trata. Recuperado de http://www.revistapueblos.org/blog/2013/01/30/el-vinculo-entre-el-vudu-y-el-delito-de-trata/

Escobar, J. y Cuervo, A. (2008). Validez de contenido y juicio de expertos: una aproximación a su utilización. Avances en Medición, 6; pp. 27-36

Flamtermesky, H. (2014). Mujer frontera. Experiencia de investigación acción participativa feminista (IAPF) con mujeres víctimas de la trata de personas. Athenea Digital: Revista de Pensamiento e Investigación Social, 14(4), pp. 389-400

Fondo de las Naciones Unidas para la infancia (2000). West and Central African Regional Office and UNICEF Nigeria Country Office. Recuperado de http://downloadily.net/docs/ child-trafficking-agency-in-nigeria.html

García de Diego, M. (2014) Jóvenes migrantes subsaharianas y trata en Andalucía: Intervención profesional desde el Trabajo Social Internacional. (Tesis Doctoral). Universidad de Granada, España

Gandarias, I. y García, N. (2014). Producciones narrativas: una propuesta metodológica para la investigación feminista. En I. M. Azkue, M. Luxán, M. Legarreta, G. Guzmán, I. Zirion, \& J. Carballo (Eds.), Otras formas de (re)conocer: Reflexiones, herramientas y aplicaciones desde la investigación feminista (pp. 97-110). Donostia-San Sebastián, España: Hegoa

Glaser, B. y Strauss, A. (1967). The Discovery of Grounded Theory: Strategies for Qualitative Research. Chicago: Aldine

Gregorio, C. (2017). Etnografiar las migraciones 'Sur' - 'Norte': la inscripción en nuestros cuerpos de representaciones de género, raza y nación. Empiria: Revista de metodología de ciencias sociales, 37 ; pp. 19-39 
Hadjab, H. (2016). Las nuevas generaciones de personas menores migrantes (Tesis doctoral). Universidad de Granada, España

Hammersley, M. y Atkinson P. (2003). Etnografía: métodos de investigación. Barcelona: Paidós

Jorge, E. y Antolínez, I. (2015). Elaboración del concepto "frontera" en las mujeres migrantes próximas a redes de trata de personas para la explotación sexual (pp. 431-453). En J.M. Gómez; S. Méndez; N. García y M.J. Cartes (Eds). Derechos humanos emergentes y periodismo. Sevilla: Universidad de Sevilla

Jorge, E., Antolínez, I. y Cordero, N. (2017). Irioweniasi. El hilo de la luna. Sevilla: Benilde

Kempadoo, K.; Sanghera, J. y Pattanaik, B. (2005). Trafficking and Prostitution Reconsidered: New Perspectives on Migration, Sex Work, and Human Rights. Boulder: Paradigm Publisher

Marcus, G. E. (2001). Etnografía en/del sistema mundo. El surgimiento de la etnografía multilocal. Alteridades, 11 (22), pp. 111-127

Merriam, S. (1988). Qualitative Research and Case Study Applications in Education. San Francisco: Jossey-Bass

MSSSI (2015). Plan Integral de Lucha contra la Trata de Mujeres y Niñas con fines de explotación sexual. Recuperado de http://www.violenciagenero.msssi.gob.es/planActuacion/planContraExplotacionSexual/docs/Plan_Integral_Trata_18_Septiembre2015_2018.pdf

Moll, L.; Amanti, C; Neff, D. y González, N. (1992). Funds of knowledge for teaching: using a qualitative approach to connect homes and classrooms. Theory into Practice, 21 (2)

Naciones Unidas (2000). Protocolo para prevenir, reprimir y sancionar la trata de personas, especialmente mujeres y niños, que complementa la Convención de las Naciones Unidas contra la Delincuencia Organizada Transnacional. Recuperado de: http://www.ohchr.org/ Documents/ProfessionalInterest/ProtocolTraffickingInPersons_sp.pdf

Nwogu, V. (2008). Human Trafficking from Nigeria and Voodoo. Any connections? La Strada International Newsletter, 9

Oficina Europea de Estadística (2015). Trafficking in Human Beings. Luxemburg: Publications Office of the European Union

Omorodion, F. (2009). Vulnerability of Nigerian secondary school to human sex trafficking in Nigeria. African Journal of Reproductive Health, 13(2), pp. 33-48 
Organización de las Naciones Unidas para la Educación, la Ciencia y la Cultura (2006). Human Trafficking in Nigeria: root causes and recommendations. Policy Paper, 14 (2), Paris

Organización Internacional para las Migraciones (2006). Migration, Human Smuggling and Trafficking from Nigeria to Europe. Oslo: International Peace Research Institute. Recuperado de http://publications.iom.int/bookstore/free/MRS23.pdf>

Programa de las Naciones Unidas para el Desarrollo (2016). Informe sobre Desarrollo Humano. Desarrollo Humanos para todos. Recuperado de http://hdr.undp.org/sites/default/ files/HDR2016_SP_Overview_Web.pdf

Rodríguez, M.V. (2014). La trata de menores entre Nigeria y España (Trabajo Fin de Grado). Universidad Internacional de La Rioja, España

Rodríguez, M.V. y Sánchez Carrillo, J.F. (2013). Derechos humanos, Religión y Supersticiones en Calabar (Nigeria). Trabajo Social Hoy, 68, pp. 69-88

Solana, J. L. (2011). La trata de seres humanos con fines de explotación sexual: análisis conceptual e histórico. En F. J. García Castaño y N. Kressova. (Coords.). Actas del I Congreso Internacional sobre Migraciones en Andalucía (pp. 915-923). Granada: Instituto de Migraciones

Stock, I. (2011). Gender and the dynamics of mobility: Reflections on African migrant mothers and "transit migration" in Morocco. Ethnic and Racial Studies, 35 (9), pp. 1577-1595

Tuwor, T. y Sossou, M.A. (2008). Gender discrimination and education in West Africa: strategies for maintaining girls in school. International Journal of Inclusive Education, 12 (4), pp. 363-379

Ugiagbe, E.O; Eghafona, K.A. y Omorogiuwa, T.B.E. (2007). An evaluation of the principles of primogeniture and inheritance laws among the Benin People of Nigeria. Journal of Family History, 32 (1), 1-12

United Nations Interregional Crime and Justice Research Institute (2003) Trafficking of Nigerian Girls to Italy. Recuperado de http://www.unicri.it/topics/trafficking_exploitation/ archive/women/nigeria_1/research/rr_prina_eng.pdf

United Nations Office on Drugs and Crime (2009, 2012, 2014, 2016). Global Report in Trafficking in Persons. Recuperado de https://www.unodc.org/unodc/data-and-analysis/glotip.html 
Van Dijk, R. (2001) Voodoo on the doorstep young Nigerian prostitutes and magic policing in the Netherlands. Africa, 71(4). 558-586

Women's Link Worldwide (2014) La trata de mujeres y niñas nigerianas: esclavitud entre fronteras y prejuicios. Recuperado de http://www2.womenslinkworldwide.org/wlw/new. php? $\operatorname{modo}=$ detalle_proyectos $\& \mathrm{dc}=72$ 